\title{
Carbon-carbon bond formation in aqueous media. Benzamidomethylation of some carbon nucleophiles
}

\author{
Ana Mateska ${ }^{a}$, Goran Stojkovića ${ }^{a}$ Bozhana Mikhova ${ }^{b}$, Kristina Mladenovska, ${ }^{c}$ and Emil \\ Popovski ${ }^{\mathrm{a}}$ * \\ ${ }^{a}$ Institute of Chemistry, Faculty of Natural Sciences \& Mathematics, Sts. Cyril and Methodius \\ University, PO Box 162, 1000 Skopje, Macedonia \\ ${ }^{b}$ Institute of Organic Chemistry with Centre of Phytochemistry, Bulgarian Academy of Sciences, \\ Akad.G.Bonchev Str. Bl.9, Sofia 1113, Bulgaria \\ ${ }^{c}$ Sts. Cyril and Methodius University, Faculty of Pharmacy, Vodnjanska 17, 1000 Skopje, \\ Macedonia \\ E-mail: emilp@iunona.pmf.ukim.edu.mk
}

\begin{abstract}
$\mathrm{C}-\mathrm{C}$ bond formation reactions between (benzamidomethyl)triethylammonium chloride (1) and some carbon nucleophiles (2a-f) were performed smoothly in aqueous media, under mild reaction conditions and ambient temperature. Mono-C-alkyl (3a, 3b, 3c) and di-C-alkyl (3d, 3e, 3f) derivatives were obtained without using a catalyst. Crystals of the products were easily isolated by simple filtration, in moderate yields and with no apparent contamination due to the formation of O-alkyl products.
\end{abstract}

Keywords: C-C Bond formation, carbon nucleophiles, aqueous medium

\section{Introduction}

Carbon-carbon bond formation is the essence of organic synthesis and provides the foundation for generating more complicated organic compounds from the simpler ones. In the latest decade, there has been increased recognition that organic reactions can proceed well in aqueous media and offer advantages over those occurring in organic solvents. Organic synthesis in water have attracted much attention, not only because unique reactivity is often observed in water but also because water can significantly shorten the synthetic route, increase product selectivity and reduce the volatile organic consumption. ${ }^{1}$

In this trend, Manabe and Kobayashi ${ }^{2}$ made a step forward in palladium-catalyzed allylic substitution with carbon nucleophiles as one of the most important and useful carbon-carbon bond-forming reactions. They disclosed a new catalytic system for substitution in aqueous 
medium. Also, the key of the catalytic system was the use of a catalytic amount of a carboxylic acid, which greatly accelerates the reactions.

Recently, similar catalyzed nucleophilic reactions were reported, but polar organic solvents were used as a medium. Allylic substitution of alcohols with some $\mathrm{C}$-nucleophiles such as $\beta$ diketones, catalyzed by $\mathrm{Pd}$ - complexes, gives an excellent result for forming new $\mathrm{C}-\mathrm{C}$ bonds. ${ }^{3}$ Pd-complexes also were found to catalyze nucleophilic benzylic substitution of benzylic esters, with high generality. ${ }^{4}$ In this reactions two different types of products were demonstrated, mono$\mathrm{C}$ and di-C substituted products. Catalytic allylation with anilines, nitrogen compounds and 1,3dicarbonyl compounds takes place with $\mathrm{Pd}$-complexes ${ }^{5}$ and $\mathrm{H}$-mont (or other solid Bronsted acid) ${ }^{6,7}$ as a catalyst. Direct substitution of alcohols with allyl-, propargyl- and alkynyl- silanes catalyzed by $\mathrm{InCl}_{3}{ }^{8,9}$ or with active methylene compounds catalyzed by Pd-complexes ${ }^{10}$ was also reported as a good synthetic approach that gives moderate to high yields of the products.

Literature data for the $\mathrm{C}-\mathrm{C}$ bond formation by the $\mathrm{S}_{N} 2$ reactions of enolate intermediates in aqueous medium ${ }^{11}$ are sparse. This motivated our research to be primary focused on investigating the possibility for building new $\mathrm{C}-\mathrm{C}$ bonds in the reaction of nucleophilic substitution in aqueous media without using a catalyst and secondary, on the synthesis of new benzamidomethyl derivatives, which may have a great application as constituent moieties for the intermediates within the synthesis of different biologically active compounds. It has already been demonstrated in the past and recently. ${ }^{12-16}$ For example, synthesis of benzamidomethyl ester, such as 2-benzamidomethyl-3-oxybutanoates which are used as intermediates in preparation of (2R,3S)-2-benzamidomethyl-3-hydroxybutanoates ${ }^{17,18}$ as chiral building blocks for synthesis of biologically active carbapenems. ${ }^{19-21}$ Also, new 1,3-diketones as synthons for preparation of new pyrazole, isooxazole or diazepine derivatives were synthesized, which had been obtained in the past and recently in the reactions with hydrazines ${ }^{22-24}$, hydroxylamine ${ }^{25}$ or in reactions with phenylenediamines, ${ }^{26-27}$ accordingly. Many of this type compounds can show different microbiological activity. Furthermore, synthesis of new benzamidomethyl cyanacetamides, as synthons for building of quinazolines, ${ }^{28}$ pyridinones, ${ }^{29}$ chromones $^{30}$ and other heterocycles ${ }^{23,24}$ has been performed.

Our previous results ${ }^{31-33}$ show that triethylamino group in (benzamidomethyl)triethylammonium chloride $\mathbf{1}$ can be easily replaced by different nucleophiles. In aqueous media these reactions were performed relatively fast, under mild conditions, giving almost pure products which can be easily isolated by simple filtration. 


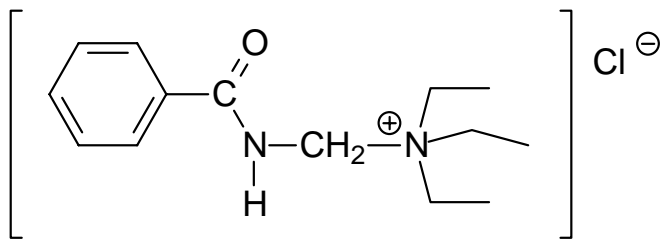

In this paper we present the formation of $\mathrm{C}-\mathrm{C}$ bonds in the reactions of nucleophilic substitution between $\mathbf{1}$ and different carbon nucleophiles, in aqueous media, without using a catalyst.

\section{Results and Discussion}

Reactions of $\mathbf{1}$ with the carbon nucleophiles as dibenzoylmethane $\mathbf{2 a}$, benzoylacetone $\mathbf{2} \mathbf{b}$, ethyl 4-nitrobenzoylacetate 2c, 1,3-indanedione 2d, 2-cyanoacetamide $2 \mathrm{e}$ and 2-cyano- $\mathrm{N}$ phenylacetamide $2 \mathbf{f}$ were performed in stirred aqueous (or $\mathrm{H}_{2} \mathrm{O}$ /acetone) media, at ambient temperature and in a presence of small quantities of triethylamine (TEA) to $\mathrm{pH} \geq 9$. Mixed solvent media $\left(\mathrm{H}_{2} \mathrm{O} /\right.$ acetone) was used in the cases where the corresponding nucleophile compound was insoluble or sparingly soluble in water. In the reactions with $\mathbf{2 a - c}$, mono-Calkylation products 3a-c were obtained (Scheme 1).<smiles>[R]C(=O)CC([R7])=O</smiles>

2

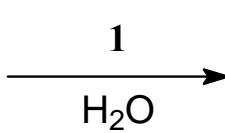

a) $\mathrm{R}^{1}=\mathrm{R}^{2}=\mathrm{Ph}$

b) $\mathrm{R}^{1}=\mathrm{CH}_{3} ; \mathrm{R}^{2}=\mathrm{Ph}$

c) $\mathrm{R}^{1}=\mathrm{OC}_{2} \mathrm{H}_{5} ; \mathrm{R}^{2}=\mathrm{C}_{6} \mathrm{H}_{4} \mathrm{NO}_{2}$<smiles>[R]C(=O)C(Br)C([R])=O</smiles>

3

\section{Scheme 1}

In the case of reactions with $\mathbf{2 d - f}$, di-C-alkylation derivatives $\mathbf{3 d - f}$ were obtained (Scheme 2 and Scheme 3). Mono-C-alkylation products in the reactions of $\mathbf{1}$ and $\mathbf{2 d}, \mathbf{2 e}, \mathbf{2 f}$ have not been isolated, despite the attempts of changing the mole ratio of reactants from 1:1 to $2: 1,3: 1$ on the 
side of the nucleophile, changing the reaction solvent mixture or by controlling the increase of $\mathrm{pH}$.<smiles>O=C1CC(=O)c2ccccc21</smiles>

2d

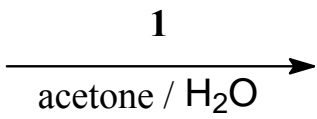

3d

\section{Scheme 2}

All products 3a-f were isolated with no apparent contamination due to the formation of Oalkyl products. $^{34}$<smiles>[R]NC(=O)CC#N</smiles>

$2(e$ or $f)$

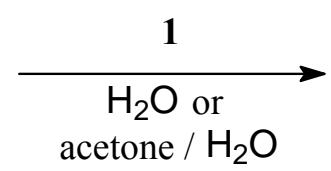

e) $\mathrm{R}^{1}=\mathrm{H}$

f) $\mathrm{R}^{1}=\mathrm{Ph}$<smiles>[R1]NC(=O)C(C#N)(C#N)C(=O)Br</smiles>

3(e or f)

\section{Scheme 3}

In the UV spectra of the reactants $\mathbf{2 a}, \mathbf{2 b}, \mathbf{2 c}$ in alcoholic solution (Table I), three different bands with maxima at $\sim 200 \mathrm{~nm}, \sim 250 \mathrm{~nm}$ and 310 (340) $\mathrm{nm}$ appeared. The peak at the longest wavelength (310 or $340 \mathrm{~nm}$ ) originates from the enol-form, and the peak at $250 \mathrm{~nm}$ could be prevalently related to keto-form and also some minor contribution of the enol-form. To illustrate the effect of mono C-benzamidomethylation on the keto-enol equilibrium, we compared the UVvisible spectra of the reactants with analogous products. In the case of $\mathbf{3 a}, \mathbf{3 b}$ and $\mathbf{3 c}$ the maxima at the longer wavelength disappeared, as a result of $\mathrm{C}-\mathrm{C}$ bond formation, which disabled or significantly inhibited the tautomeric enol-form of the products. Without enol-form, newly formed monobenzamidomethyl products considerably lose nucleophilic nature and further reaction with $\mathbf{1}$ does not occur. Good example for this is UV spectra of $\mathbf{2 b}$ and $\mathbf{3 b}$ presented in Figure 1. Monobenzamidomethyl derivative of 2d was not isolated to measure UV-VIS spectrum. 
Figure 1. UV-visible spectra of $\mathbf{2 b}$ and $\mathbf{3 b}$.

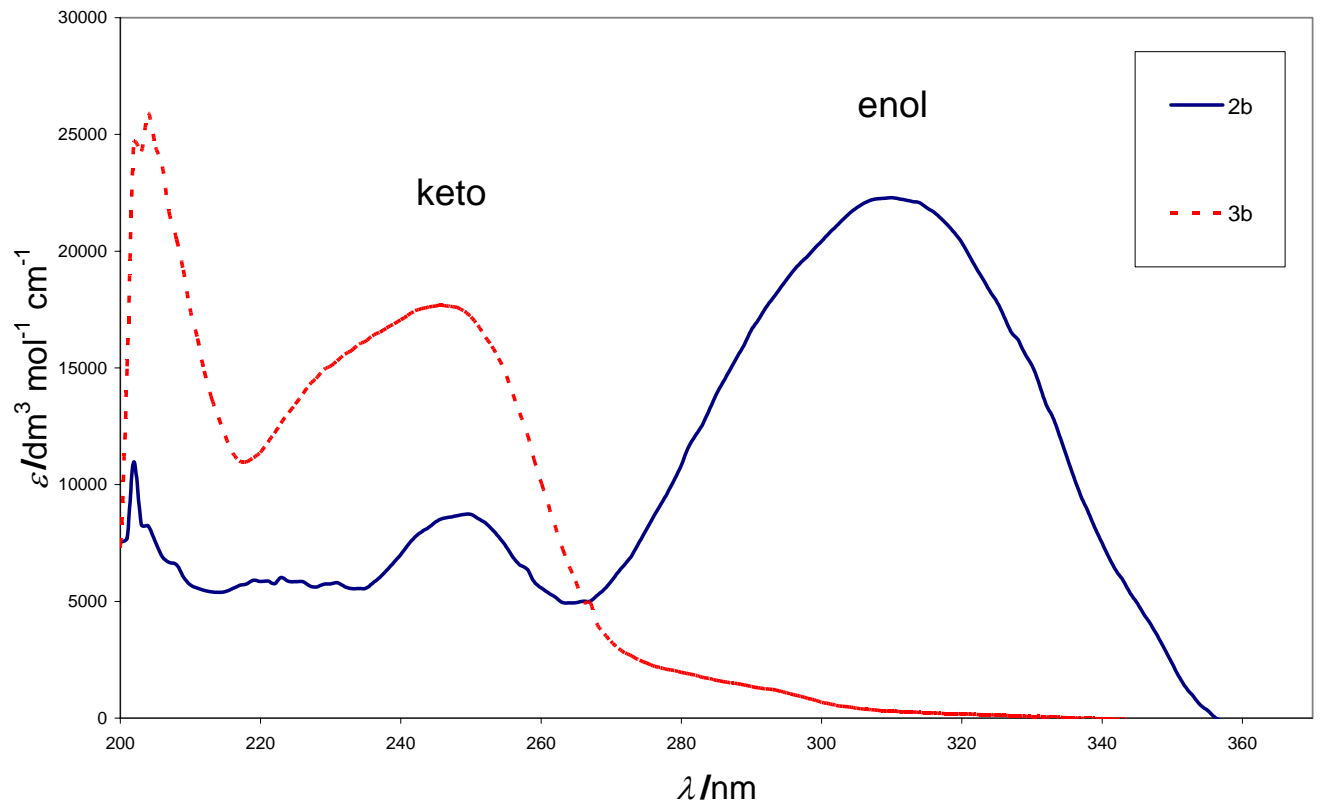

Table 1. UV data for compounds $\mathbf{2 a - f}$ and 3a-f

\begin{tabular}{cccccccc}
\hline React. & $c / \mathrm{mol} \mathrm{dm}^{-3}$ & $\lambda_{\max } / \mathrm{nm}$ & $\log \{\varepsilon\}$ & Product & $c / \mathrm{mol} \mathrm{dm}^{-3}$ & $\lambda_{\max } / \mathrm{nm}$ & $\log \{\varepsilon\}$ \\
\hline 2a & $2.20 \cdot 10^{-5}$ & 206 & 5.04 & 3a & $5.85 \cdot 10^{-5}$ & 206 & 4.51 \\
& & 248 & 5.09 & & & 248 & 4.55 \\
& & 342 & 4.46 & & & $342(\mathrm{sh})$ & 0.53 \\
2b & $2.12 \cdot 10^{-5}$ & 202 & 4.04 & 3b & $6.67 \cdot 10^{-5}$ & 202 & 4.39 \\
& & 246 & 3.93 & & & 246 & 4.25 \\
& & 310 & 4.35 & & & $310(\mathrm{sh})$ & 2.48 \\
2c & $1.20 \cdot 10^{-4}$ & 202 & 4.09 & 3c & $5.43 \cdot 10^{-5}$ & 202 & 4.38 \\
& & -- & -- & & & 229 & 4.09 \\
& & 260 & 4.04 & & & 260 & 4.12 \\
& & 311 & 3.92 & & & $311(\mathrm{sh})$ & 3.14 \\
2d & $3.42 \cdot 10^{-5}$ & 200 & 3.98 & 3d & $4.44 \cdot 10^{-5}$ & 204 & 4.56 \\
& & 224 & 4.54 & & & 227 & 4.80 \\
& & 249 & 4.01 & & & $249(\mathrm{sh})$ & 4.38 \\
2e & $1.91 \cdot 10^{-3}$ & 204 & 2.83 & 3e & $1.48 \cdot 10^{-5}$ & 202 & 4.77 \\
& & $229(\mathrm{sh})$ & 1.99 & & & 229 & 4.66 \\
& & 250 & 1.53 & & & $250(\mathrm{sh})$ & 4.24 \\
2f & $7.39 \cdot 10^{-6}$ & 201 & 4.65 & 3f & $1.38 \cdot 10^{-5}$ & 202 & 4.88 \\
& & 243 & 4.44 & & & 233 & 4.69 \\
\hline
\end{tabular}

$[\varepsilon]=\mathrm{dm}^{3} \mathrm{~mol}^{-1} \mathrm{~cm}^{-1} ;(\mathrm{sh})-$ shoulder 
In the ${ }^{1} \mathrm{H}-\mathrm{NMR}$ spectra of monobenzamidomethyl derivatives $\mathbf{2 a}, \mathbf{2} \mathbf{b}$ and $\mathbf{2 c}$, the signal from $\mathrm{CH}$ appears as triplet at $6.1 \mathrm{ppm}, 5.06 \mathrm{ppm}$ and $5.03 \mathrm{ppm}$, respectively. As expected, this signal is absent in the ${ }^{1} \mathrm{H}-\mathrm{NMR}$ spectra of dibenzamidomethyl derivatives.

The reason why $\mathbf{2 a}, \mathbf{2 b}$ and $\mathbf{2 c}$ gives monobenzamidomethyl derivatives, and $\mathbf{2 d}$ dibenzamidomethyl derivative, probably was due to the differences in the acidity of the nucleophilic compounds: $\mathrm{pKa} \mathbf{2 a}=15.2,{ }^{35} 13.4,{ }^{36} 13.3 ;^{37} \mathrm{pKa} \mathbf{2} \mathbf{b}=14.2,{ }^{35,37} 12.3 ;^{38} \mathrm{pKa} \mathbf{2 c}=$ $11 ;^{37}$ pKa $2 \mathbf{d}=7.2,{ }^{39-41} 7.32-7.38,{ }^{42} 8.95 .^{37}$ More acidic the nucleophile is (e.g. 2d) greater the activity of the firstly formed mono-C-alkylated derivative which is most likely existing in enolform. Therefore, the reaction proceeds immediately, by attaching the second nucleophile group to the salt, thus forming di-C-alkylated derivative. For this reason, monobenzamidomethyl derivative of $\mathbf{2 d}$ (which might be expected as intermediate) was not isolated, regarding this or similar reaction conditions. Weaker acids $\mathbf{2 a}, \mathbf{2 b}$ and $\mathbf{2 c}$ give monobenzamidomethyl derivatives as much weaker nucleophiles.

The last type of the carbon nucleophiles that were included in this research were the $\beta$ ketonitriles. In the reaction of $\mathbf{1}$ with $\mathbf{2 e}, \mathbf{2 f}$, di-C-alkyl derivatives were isolated. $\mathrm{pKa} \mathbf{2 e}=\mathbf{-}$ $1.27^{37}$ and pKa $\mathbf{2 f}=-1.03^{37}$ show similarity with the reaction of $\mathbf{1}$ with $\mathbf{2 d}$. Most likely, firstly formed monobenzamidomethyl derivatives are still good nucleophiles and the kinetics of the reaction is so high, that di-C-alkylated products are the only possible derivatives synthesized under this reaction conditions.

\section{Conclusions}

This research demonstrates that $\mathrm{C}-\mathrm{C}$ bond forming processes can occur in aqueous media in the reaction between quaternary ammonium salt $\mathbf{1}$ and different carbon nucleophiles. Depending on the acidity of the nucleophilic compounds mono- and di-C-alkylation products were isolated. More acidic the nucleophile is, the process of second deprotonation is predominant, giving diproducts without any possibility of isolating mono products under this or similarly modified reaction conditions. During this research some new benzamidomethyl derivatives were synthesized, which can be used as intermediates for the synthesis of potential biologically active compounds.

\section{Experimental Section}

General Procedures. Melting points were determined with Reichert heating plate and were uncorrected. Carbon-hydrogen elemental analysis was carried out with Coleman Model 33. Nitrogen elemental analysis was carried out by the Dumas method. Mass spectra were measured on a API QSTAR PULSAR I. NMR spectra were recorded on a Bruker 400 using DMSO- $d_{6}$ and $\mathrm{CDCl}_{3}$ as solvents. Infrared spectra were measured on a Perkin-Elmer System 2000 FT IR, by 
the method of $\mathrm{KBr}$ pellets. UV-visible spectra were recorded on a Varian Cary 50 Spectrophotometer in $1 \mathrm{~cm}$ quartz cells. All the reagents and solvents were obtained from commercial sources and were used without further purification.

(Benzamidomethyl) triethylammonium chloride $\mathbf{1}$ was obtained in manner described in previous work. $^{32}$

\section{General procedure for synthesis of 3a-f}

A water solution of $\mathbf{1}$ was added to a water solution ( or $_{2} \mathrm{O}$ /acetone solution in all cases needed) of 2 with TEA to $\mathrm{pH}=9-11$. The mixture was stirred for $1 \mathrm{~h}$ at ambient temperature and all the products 3a-f were collected by filtration.

$N$-(2-Benzoyl-3-oxo-3-phenylpropyl) benzamide (3a). 1 (0.9 g, $3.1 \mathrm{mmol}), 2 \mathrm{a}(0.5 \mathrm{~g}, 2.1$ $\mathrm{mmol})$ and TEA $(0.3 \mathrm{ml}, \mathrm{pH}=9)$ in acetone/water. Yellow crystals, recrystallized from acetone. Mp: $157-158{ }^{\circ} \mathrm{C}$ (lit. ${ }^{43} 158-160{ }^{\circ} \mathrm{C}$ ); Yield 53.4 \% Anal.Calcd. for $\mathrm{C}_{23} \mathrm{H}_{19} \mathrm{NO}_{3}$ : C, $77.3 \%$; $\mathrm{H}, 5.4$ \%; N, $3.9 \%$. Found: C, $77.1 \%$; H, $5.6 \%$; N, $3.8 \%$. FTIR(KBr) $/ \mathrm{cm}^{-1}$ : 3428(s) and 3398(s) $(\mathrm{vNH}) ; 1697(\mathrm{vs}), 1684(\mathrm{vs})$ and $1670(\mathrm{vs})(\mathrm{vCO}) ; 1651(\mathrm{vs})$ Amide I; $1529(\mathrm{vs})$ Amide II; ${ }^{1} \mathrm{H}-$ NMR(400 MHz; DMSO-d 6 ) $/$ ppm $8.73(1 \mathrm{H}, \mathrm{t}, J 5.6, \mathrm{NH}) ; 8.04-7.39(15 \mathrm{H}, 3 \mathrm{Ph}) ; 6.08(1 \mathrm{H}, \mathrm{t}, J=$ 6.4, $\mathrm{CH}) ; 3.89\left(2 \mathrm{H}, \mathrm{t}, J=6.0, \mathrm{NHCH}_{2}\right) ;{ }^{13} \mathrm{C}-\mathrm{NMR}(100 \mathrm{MHz}$; DMSO-d 6 )/ppm $195.4 \mathrm{C}=\mathrm{O} ; 166.9$ $\mathrm{CONH}, 68.4 \mathrm{CH}_{2} ; 54.8 \mathrm{CH}$; Aromatic: 135.6; 134.0; 133.9; 131.2; 129.0; 128.4; 128.2 and 127.1. MS (ESI, positive ion mode): $\mathrm{m} / \mathrm{z} 358.1(\mathrm{M}+\mathrm{H})^{+}$.

$\mathrm{N}$-(2-Benzoyl-3-oxobutyl) benzamide (3b). $1(1.5 \mathrm{~g}, 6.0 \mathrm{mmol}), \mathbf{2 b}(0.45 \mathrm{~g}, 3.0 \mathrm{mmol})$ and TEA $(0.1 \mathrm{ml}, \mathrm{pH}=9)$ in water. White crystals, recrystallized from acetone. Mp: $105-107^{\circ} \mathrm{C}$ (lit. ${ }^{43}$ 108-109 ${ }^{\circ} \mathrm{C}$ ); Yield $33.9 \%$ Anal.Calcd. for $\mathrm{C}_{18} \mathrm{H}_{17} \mathrm{NO}_{3}: \mathrm{C}, 73.2 \%$; H, 5.8\%; N, $4.5 \%$. Found: $\mathrm{C}$, $72.9 \%$; H, $6.1 \%$; N, $4.6 \%$. FTIR(KBr) $/ \mathrm{cm}^{-1}: 3430(\mathrm{~s})$ and 3324.0(m) $(\mathrm{vNH}) ; 1724(\mathrm{vs})$ and 1661(s) (vCO);1637(vs) Amide I; 1534(s) Amide II; ${ }^{1} \mathrm{H}-\mathrm{NMR}\left(400 \mathrm{MHz} ; \mathrm{CDCl}_{3}\right) / \mathrm{ppm}$ 8.14-7.42 $(10 \mathrm{H}, 2 \mathrm{Ph}) ; 6.86(1 \mathrm{H}, \mathrm{br}(\mathrm{t}), \mathrm{NH}) ; 5.06(1 \mathrm{H}, \mathrm{t}, J 6.0, \mathrm{CH}) ; 4.10\left(1 \mathrm{H}, \mathrm{m}, \mathrm{NHCH}_{2}\right) ; 3.89(1 \mathrm{H}, \mathrm{m}$, $\left.\mathrm{NHCH}_{2}\right) ; 2.26\left(3 \mathrm{H}, \mathrm{s}, \mathrm{CH}_{3}\right){ }^{13} \mathrm{C}-\mathrm{NMR}\left(100 \mathrm{MHz} ; \mathrm{CDCl}_{3}\right) / \mathrm{ppm} 204.2 \mathrm{C}=\mathrm{O} ; 196.7 \mathrm{C}=\mathrm{O} ; 168.5$ CONH; 61.6 $\mathrm{CH}_{2}$; 39.5 CH; 30.5 $\mathrm{CH}_{3}$; Aromatic: 136.5, 134.9, 134.5, 132.4, 129.8, 129.6, 129.3 and 127.6. MS (EI POS. Temp): $\mathrm{m} / \mathrm{z} 294.8 \mathrm{M}^{+}$.

Ethyl 2-(4-nitrophenyl)-3-[(phenylcarbonyl)amino]propanoat (3c). 1 (1 g, $3.7 \mathrm{mmol}), 2 \mathrm{c}$ $(0.67 \mathrm{~g}, 2.8 \mathrm{mmol})$ and TEA $(0.5 \mathrm{ml}, \mathrm{pH}=9)$ in water. Yellow crystals, recrystallized from acetone. Mp: 158-160 ${ }^{\circ} \mathrm{C}$; Yield $37 \%$.Anal.Calcd. for $\mathrm{C}_{19} \mathrm{H}_{18} \mathrm{~N}_{2} \mathrm{O}_{6}$ : C, $61.6 \%$;, $4.9 \%$; N, 7.6 \%. Found: C, $61.4 \%$; H, $4.7 \%$; , $7.5 \%$. FTIR(KBr) $/ \mathrm{cm}^{-1}$ : 3430(s) (vNH); 1743(vs) (vCOOC);1686(s) (vCO); 1656(s) Amide I; 1527(vs) Amide II; ${ }^{1} \mathrm{H}-\mathrm{NMR}(400 \mathrm{MHz}$; DMSO$\left.\mathrm{d}_{6}\right) / \mathrm{ppm} 8.71(1 \mathrm{H}, \mathrm{t}, J=6.0, \mathrm{NH}) ; 8.34-7.41(9 \mathrm{H}, 2 \mathrm{Ph}) ; 5.05(1 \mathrm{H}, \mathrm{t}, J=7.2, \mathrm{CH}) ; 4.08(2 \mathrm{H}, \mathrm{q}, J$ $\left.=7.2, \mathrm{CH}_{2} \mathrm{CH}_{3}\right) ; 3.84\left(2 \mathrm{H}, \mathrm{t}, J=6.0, \mathrm{NCH}_{2}\right) ; 1.06\left(3 \mathrm{H}, \mathrm{t}, J 7.2, \mathrm{CH}_{3}\right) ;{ }^{13} \mathrm{C}-\mathrm{NMR}(100 \mathrm{MHz}$; DMSO-d $\left.{ }_{6}\right) / \mathrm{ppm} 193.8 \mathrm{C}=\mathrm{O} ; 167.9 \mathrm{C}=\mathrm{O} ; 166.8 \mathrm{C}=\mathrm{O} ; 61.3 \mathrm{CH}_{2} ; 53.2 \mathrm{CH} ; 38.6 \mathrm{CH}_{2} ; 13.7 \mathrm{CH}_{3}$; Aromatic: 150.2, 140.4, 133.9, 131.3, 129.8, 128.2, 127.1 and 124.0. MS (ESI, positive ion mode): $\mathrm{m} / \mathrm{z} 371.1(\mathrm{M}+\mathrm{H})^{+}$.

$N$-[(1,3-Dioxo-2,3-dihidro-1H-inden-2-yl)methyl]benzamide (3d). 1 ( $0.9 \mathrm{~g}, 3.3 \mathrm{mmol}), \mathbf{2 d}(0.3$ $\mathrm{g}, 2.3 \mathrm{mmol})$ and TEA $(0.1 \mathrm{ml}, \mathrm{pH}=9)$ in water/acetone. Purple crystals, recrystallized from 
acetone. Mp: $200-203{ }^{\circ} \mathrm{C}$ Yield 42.2 \%. Anal.Calcd. for $\mathrm{C}_{25} \mathrm{H}_{20} \mathrm{~N}_{2} \mathrm{O}_{4}: \mathrm{C}, 72.8 \%$; $\mathrm{H}, 4.9 \%$; , $6.8 \%$. Found: C, $72.7 \%$; H, $5.1 \%$; N, $6.9 \%$. FTIR(KBr $/ \mathrm{cm}^{-1}: 3336(\mathrm{~m})$ and 3278(m) $(v \mathrm{NH})$; 1743(m) and 1705(vs) (vCO); 1653(s) Amide I; 1537(s) Amide II; ' ${ }^{\mathrm{H}-N M R}(400 \mathrm{MHz}$; DMSO$\left.\mathrm{d}_{6}\right) / \mathrm{ppm} 8.64(2 \mathrm{H}, \mathrm{t}, J=5.6,2 \mathrm{xNH}) ; 7.91-7.37(14 \mathrm{H}, \mathrm{Ar}) ; 3.73(4 \mathrm{H}, \mathrm{d}, J=6.0,2 \mathrm{xNH}) ;{ }^{13} \mathrm{C}-$ NMR(100 MHz; DMSO-d 6 )/ppm 199.3 C=O; 166.9 CONH; $68.4 \mathrm{CH}_{2} ; 58.4 \mathrm{C}$; Aromatic: 141.5 , 135.8, 133.9, 131.3, 128.2, 127.1 and 122.9. MS (ESI, positive ion mode): $\mathrm{m} / \mathrm{z} 413.1(\mathrm{M}+\mathrm{H})^{+}$.

$\mathrm{N}$-(3-Amino-2-cyano-3oxopropyl)benzamide (3e). 1 (1 g, $3.7 \mathrm{mmol}), 2 \mathrm{e}(0.3 \mathrm{~g}, 2.9 \mathrm{mmol})$ and TEA $(0.1 \mathrm{ml}, \mathrm{pH}=9)$ in water. White crystals recrystallized from water. Mp: $166-169^{\circ} \mathrm{C}$; Yield $24 \%$. Anal.Calcd. for $\mathrm{C}_{19} \mathrm{H}_{18} \mathrm{~N}_{4} \mathrm{O}_{3}: \mathrm{C}, 65.1 \%$; H, $5.2 \%$; N, $16.0 \%$. Found: C, $64.9 \%$; H, $5.3 \%$; $\mathrm{N}, 15.8 \%$. FTIR(KBr) $/ \mathrm{cm}^{-1}: 3423(\mathrm{~s}), 3387(\mathrm{~s}), 3332(\mathrm{~s})$ and 3277(s) $(\mathrm{vNH}) ; 2246(\mathrm{w})(\mathrm{vCN})$; 1681(vs) and 1654(vs) Amide I; 1536(vs) Amide II; ${ }^{1} \mathrm{H}-\mathrm{NMR}(400 \mathrm{MHz}$; DMSO-d 6 )/ppm 8.68 $(2 \mathrm{H}, \mathrm{t}, J=6.5,2 \mathrm{xNHCO}) ; 7.77-7.37\left(12 \mathrm{H}, \mathrm{Ar}+\mathrm{NH}_{2}\right) ; 3.82 \mathrm{dd}$ and $3.72 \mathrm{dd}(4 \mathrm{H}, \mathrm{AB}$ from ABX, $J 14.0$ and 6.5, $\left.2 \mathrm{xNHCH}_{2}\right) ;{ }^{13} \mathrm{C}-\mathrm{NMR}\left(100 \mathrm{MHz}\right.$; DMSO- $\left.\mathrm{d}_{6}\right) / \mathrm{ppm} 167.2 \mathrm{C}=\mathrm{O} ; 166.8 \mathrm{C}=\mathrm{O} ; 118.4$ $\mathrm{CN} ; 51.8 \mathrm{CH}_{2}$; $41.5 \mathrm{CCN}$; Aromatic: 133.8, 131.6, 128.4, 127.1; MS (ESI, positive ion mode) : $\mathrm{m} / \mathrm{z} 351.1(\mathrm{M}+\mathrm{H})^{+}$.

$N$-(3-Anilino-2-cyano-3-oxopropul)benzamide (3f). 1 ( $0.9 \mathrm{~g}, 3.14 \mathrm{mmol}), 2 \mathrm{f}(0.5 \mathrm{~g}, 1.7 \mathrm{mmol})$ and TEA $(0.2 \mathrm{ml}, \mathrm{pH}=11-12)$ in water/acetone. White crystals recrystallized from ethanol. Mp: $178-181^{\circ} \mathrm{C}$; Yield $32.6 \%$. Anal.Calcd. for $\mathrm{C}_{25} \mathrm{H}_{22} \mathrm{~N}_{4} \mathrm{O}_{3}: \mathrm{C}, 69.6 \%$;, $5.2 \% ; \mathrm{N}, 14.3 \%$. Found: C, $69.5 \%$; H, $5.0 \%$; N, $14.5 \%$. FTIR(KBr) $/ \mathrm{cm}^{-1}: 3334(\mathrm{~s})$ and 3279(m/sh) $(v \mathrm{NH}) ; 2254(\mathrm{w})$ (vCN); 1699(s) and 1654(vs)Amide I; 1539(vs) Amide II; ${ }^{1} \mathrm{H}-\mathrm{NMR}\left(400 \mathrm{MHz}\right.$; DMSO-d $\left.\mathrm{d}_{6}\right) / \mathrm{ppm}$ $10.17(1 \mathrm{H}, \mathrm{s}, \mathrm{NHPh}) ; 8.91\left(2 \mathrm{H}, \mathrm{t}, J=6.3, \mathrm{NHCH}_{2}\right) ; 7.88-7.13(15 \mathrm{H}, 3 \mathrm{Ph}) ; 4.12 \mathrm{dd}$ and $4.02 \mathrm{dd}$ (4H, AB from ABX, $J=14.0$ and $6.3,2 \mathrm{xNHCH}_{2}$ ); ${ }^{13} \mathrm{C}-\mathrm{NMR}(100 \mathrm{MHz}$; DMSO-d 6 )/ppm 167.3 $\mathrm{C}=\mathrm{O} ; 163.5 \mathrm{C}=\mathrm{O} ; 117.9 \mathrm{CN} ; 53.0 \mathrm{CH}_{2}$; $41.3 \mathrm{CCN}$; Aromatic: 138.0, 133.7, 131.5, 128.3, 128.2, 127.3, 124.3 and 121.4; MS (ESI, positive ion mode) : $\mathrm{m} / \mathrm{z} 427.5(\mathrm{M}+\mathrm{H})^{+}$.

\section{Acknowledgements}

We are grateful and appreciate the technical support and hospitality from the Faculty of Chemistry and Mineralogy at the University of Leipzig, especially to Prof. Dr. Evamarie HeyHawkins.

\section{References and Notes}

1. Chao-Jun, L. Chem. Rev. 2005, 105, 3095.

2. Manabe, K.; Kobayashi, S. Org. Lett. 2003, 5, 3241.

3. Kayaki, Y.; Koda, T.; Ikariya, T. J. Org. Chem. 2004, 69, $2595-$.

4. Kuwano, R.; Kondo, Y.; Matsuyama, Y. J. Am. Chem. Soc. 2003, 125, 12104. 
5. 5. Ozawa, F.; Okamoto, H.; Kawagishi, S.; Yamamoto, S.; Minami, T.; Yoshifuji, M. J. Am. Chem. Soc. 2002, 124, 10968.

6. Motokura, K.; Nakagiri, N.; Mizugaki, T.; Ebitani, K.; Kaneda, K. J. Org. Chem. 2007, 72, 6006.

7. Sanz, R.; Martinez, A.; Miguel, D.; Alvarez-Gutierrez, M. J.; Rodrigez, F. Adv. Synh. Catal. 2006, 348, 1841.

8. Saito, T.; Yasuda, M.; Baba, A. Synlett 2005, 11, 1737.

9. Yasuda, M.; Saito, T.; Ueba, M.; Baba, A. Angew. Chem. Int. Ed. 2004, 43, 1414.

10. Kimura, M.; Mukai, R.; Tanigawa, N.; Tanaka, S.; Tamaru, Y. Tetrahedron 2003, 59, 7767.

11. Cerichelli, G.; Cerritelli, S.; Chiarini, M.; De Maria, P.; Fontana, A. Chem. Eur. J. 2002, 8 (22), 5204.

12. Tiwari, K. A.; Singh, K. V.; Bajpai, A.; Shukla, G.; Singh, S.; Mishra, K. A. Eu. Jour. of Med. Chem. 2007, 42 (9), 1234.

13. Zlotin, G.S.; Sharova, V.I.; Luk'yanov, A.O. Rus. Chem. Bul. 1996, 45(7), 1680.

14. Csomós, P.; Fodor, L.; Bernáth, G.; Csámpai, A.; Sohár, P. Tetrahedron 2008, 648646.

15. Csomós, P.; Fodor, L.; Bernáth, G.; Csámpai, A.; Sohár, P. Tetrahedron 2009, 651475.

16. Mai, A.; Artico, M.; Valente, S.; Cerbara, I.; Befani, O.; Turini, P.; Vedova, D. L. Agostinelli, E. ARKIVOC 2004 (v), 32.

17. Noyori, R.; Kitamura, M.; Ohkuma, T. PNAS 2004, 101 (15), 5356.

18. Cesarotti, E.; Rimoldi, I.; Spalluto, P.; Demartin, F. Tetrahedron:Asymmetry 2007, 18, 1278.

19. Shimoda, K.; Kubota, N.; Hamada, H.; Kobayashi, T.; Hamada, H.; Shafi, S. M.; Nakajima, N. Biochemistry Insights 2009, 2, 5.

20. Shimoda, K.; Kubota, N.; Hamada, H.; Hamada, H. Tetrahedron Lett. 2006, 47, 1541.

21. Kumobayashi, H.; Miura, T.; Sayo, N.; Saito, T. Jour. Synt. Org. Chem. Japan 1999, 57 (5), 387.

22. Aggarwal, R.; Sumran, G.; Kumar, R.; Singh, S. P. ARKIVOC 2007, (xv), 292.

23. El-Saghier, A. M. M.; Naili, M. B.; Rammash B. K.; Saleh, N. A.; Kreddan, K. M. ARKIVOC 2007, (xvi), 83.

24. El-Faraegy, A. F.; Ghonium, A. A. ARKIVOC 2008, (xiii), 278.

25. Razus, C. A.; Birzan, L.; Nae, S.; Lehadus, L. O.; Pavel, C.; Costan, O. ARKIVOC 2005, $(x)$, 71.

26. Kumar, R.; Joshi, C. Y. ARKIVOC 2007, (xiii), 142.

27. Tsoleridis, A. C.; Pozarentzi, M.; Mitkidou, S.; Stephanidou-Stephanatou, J. ARKIVOC 2008, (xv), 193.

28. Parkhomenko, O. O.; Kovalenko, M. S.; Chernykh, P. V.; Osolodchenko, P. T. ARKIVOC 2005, (viii), 82 .

29. Katritzky, R. A.; Silina, A.; Tymoshenko, O.D.; Qiu, G.; Nair, K. S.; Steel, J.P. ARKIVOC 2001, (vii), 138.

30. Ibrahim, A. M. ARKIVOC 2008, (xvii), 192.

31. Popovski, E.; Bogdanov, J.; Najdoski, M.; Hey-Hawkins, E. Molecules 2006, 11, 279. 
32. Popovski, E.; Klisarova, L.; Vikic-Topic, D. Molecules 2000, 5, 927.

33. Popovski, E.; Klisarova, L.; Vikic-Topic, D. Synth. Commun. 1999, 29, 3451.

34. Clark, H. J.; Miller, M. J. J. Chem. Soc., Chem. Commun. 1977, 64.

35. Saad, R. G.; Naoum, M. M.; Rizk, A. H. Can. J. Chem. 1989, 67, 284.

36. Arnett, M. E.; DePalma, V.; Maroldo, S.; Small, S. L.; Pure \& Appl. Chem. 1979, 51, 131.

37. Note: $\mathrm{pKa}$ values for each compound were obtained from SciFind Scholar - calculated using Advanced Chemistry Development (ACD/Labs) Software V8.14 for Solaris (1994-2008 $\mathrm{ACD} / \mathrm{Labs}$ ).

38. Le, T. H. Q.; Umetani, S.; Matsui, M. J. Chem. Soc., Dalton Trans. 1997, 3835.

39. Tu, S.; Jiang, B.; Jia, R.; Zhang, J.; Zhang, Y. Tetrahedron Lett. 2007, 48, 1369.

40. Tu, S.; Jiang, B.; Jiang, H.; Zhang, Y.; Jia, R.; Zhang, J.; Shao, Q.; Li, C.; Zhou, D.; Cao, L. Tetrahedron 2007, 63, 5406.

41. Tu, S.; Wu, S.; Yan, S.; Hao, W.; Zhang, X.; Cao, X.; Han, Z.; Jiang, B.; Shi, F.; Xia, M.; Zhou, J. J. Comb. Chem. 2009, 11, 239.

42. Aydemir, F. M.Sc. Thesis, Gazi University, 1991.

43. Monti, L. Gazz. Chim. Ital. 1930, 60, 39. 